\title{
Archéopages
}

Archéopages

Archéologie et société

45 | 2017

De la terre au pot

\section{Les ratés de cuisson du haut Moyen Âge de l'atelier de Saran}

Témoins des évolutions d'une chaîne opératoire

The misfires of the Early Middle Ages of Saran's workshop. Witnesses of changes in an operational chain

Los fracasos de la cocción de la Alta Edad Media en el taller de Saran.

Testimonios de la evolución de una cadena operatoria

Sébastien Jesset, Jérôme Bouillon, Coline Lejault, Anne Bocquet-Liénard, avec la collaboration de François Capron, Amélie Laurent-Dehecq et Sébastien Millet

\section{OpenEdition}

Journals

Édition électronique

URL : https://journals.openedition.org/archeopages/3220

DOI : 10.4000/archeopages.3220

ISSN : 2269-9872

Éditeur

INRAP - Institut national de recherches archéologiques préventives

Édition imprimée

Date de publication : 1 mars 2018

Pagination : 82-95

ISSN : 1622-8545

Référence électronique

Sébastien Jesset, Jérôme Bouillon, Coline Lejault, Anne Bocquet-Liénard, avec la collaboration de François Capron, Amélie Laurent-Dehecq et Sébastien Millet, "Les ratés de cuisson du haut Moyen Âge de l'atelier de Saran », Archéopages [En ligne], 45 | 2017, mis en ligne le 01 janvier 2020, consulté le 03 juin 2021. URL : http://journals.openedition.org/archeopages/3220 ; DOI : https://doi.org/ 10.4000/archeopages.3220 


\section{Les ratés de cuisson \\ du haut Moyen Âge de l'atelier \\ de Saran \\ Témoins des évolutions d'une chaîne opératoire}

Sébastien Jesset Orleans Métropole, Póle d'Archeologie

Jérôme Bouillon Inrap

Coline Lejault Département du Loiret, servicede darcheologie preventive

Anne Bocquet-Liénard CNRs, Uмк 6273, « Craham "

avec la collaboration de François Capron Inrap, Amélie Laurent-Dehecq Département tu Loiret service de larch heloggie preventive et Sébastien Millet ${ }^{\text {Irrap }}$

\section{2}

1. Saran : la Médecinerie la Hutte : 24 fours $\mathrm{VI}^{\mathrm{e}}$ - $\mathrm{IX}^{\mathrm{e}}$ siècle

2. Saran : la Guignace: 11 fours VI ${ }^{\mathrm{e}}$-IX ${ }^{\mathrm{e}}$ siècle

3. Saran : Voie Nouvelle :

1 four VIII ${ }^{\mathrm{e}}$ siècle

4. Saran : le Bourg

1 four $\mathrm{X}^{\mathrm{e}}$ siècle

5. Saran : ZAC du Champ

Rouge : ratés de

cuisson $\mathrm{IX}^{\mathrm{e}}$ siècle

6. Saran : ZAC

des Vergers : 4 fours VII $^{\mathrm{e}}$-IX ${ }^{\mathrm{e}}$ siècle

7. Saran : ZAC Portes du Loiret/le Mesnil : 3 fours $\mathrm{VIII}^{\mathrm{e}}-\mathrm{X}^{\mathrm{e}}$ siècle

8. Ingré : Selliers :

3 fours $\mathrm{VI}^{\mathrm{e}}-\mathrm{VII}^{\mathrm{e}}$ siècle

9. Saran : la Montjoie :

1 four non daté

surface

diagnostiquée

ث secteur ou unité

de production

- four isolé

_ voie antique

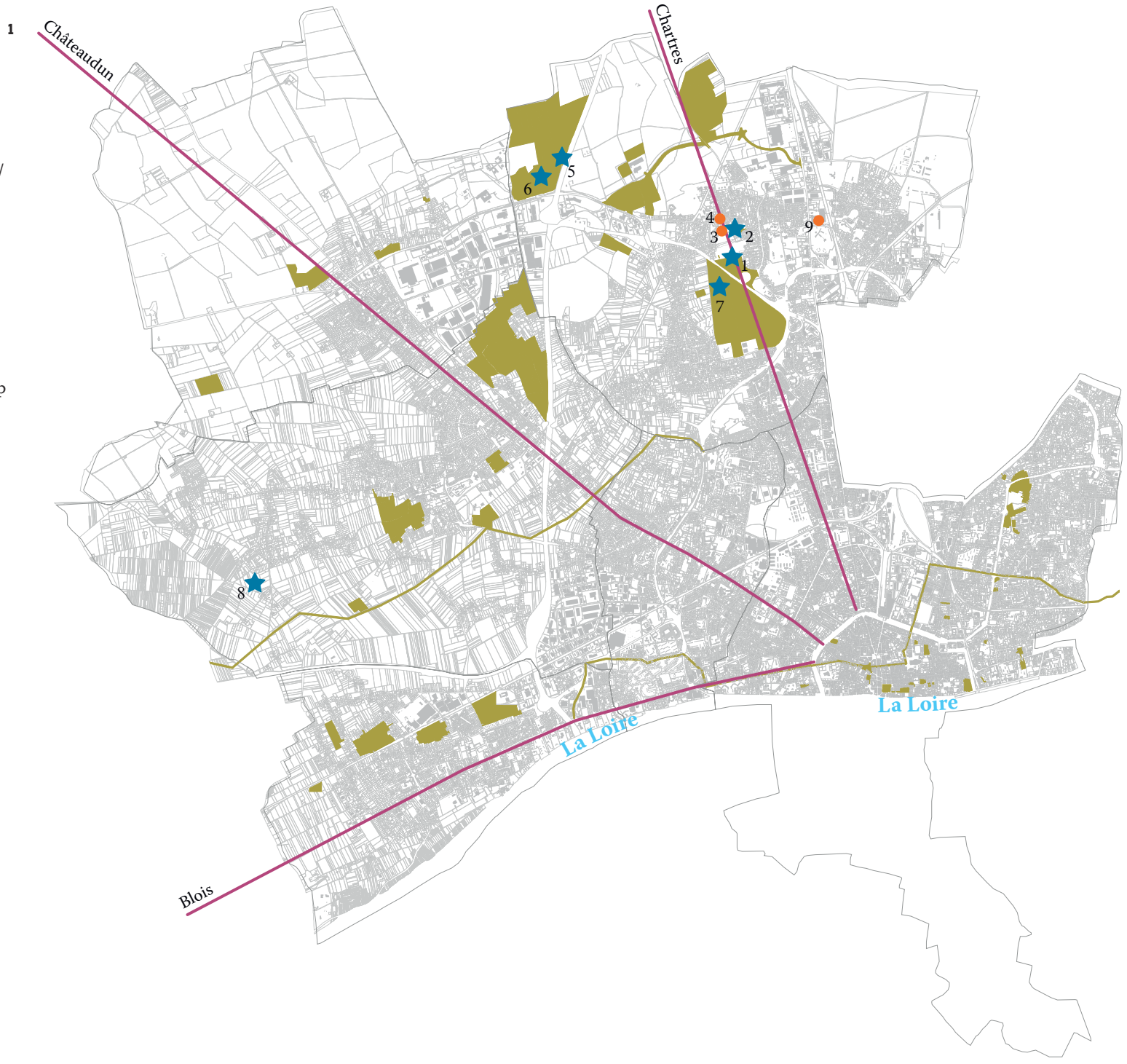


1. L'artisanat potier au haut

e ( $v 1^{e}-x^{e}$ siécle)

dans le quart nord-ouest de

l'agglomération orléanaise.

La zone du u Bourg sud "

à Saran regroupe les vestiges

découverts aux lieux-dits

de la Médecinerie/la Hutte,

laZAC Portes du Loiret/

le Mesnil, le Bourg, la Source

Saint Martin/la Voie Nouvelle

et la Guignace. Les zones

de la "ZAC des Vergers "

et du " Champ Rouge 》"

se situent en limite nord

de la commune. La zone

de ul la Montjoie u vers

l'est correspond à la mise

au jour en 1901 d'un four

de tuilier non daté.
1. Trente-huit analyses réalisées par ICP-AES par Lætitia Birée

(Normandie université,

Craham), à l'aide

d'un spectromètre

d'émission atomique.

2. Cet aspect de la chaîne

opératoire serait encore

mieux appréhendé

par des analyses

complémentaires,

en microscopie optique

par exemple.
La commune de Saran, située à $6 \mathrm{~km}$ au nord d'Orléans, est remarquable par la zone d'artisanat potier qui a pu s'y développer durant tout le haut Moyen Âge. La coexistence de facteurs naturels et économiques a largement contribué à l'essor de cette activité spécifique sur ce territoire : une argile de qualité, accessible et abondante ; un combustible pérenne avec une localisation en lisière d'un massif forestier d'envergure (forêt domaniale d'Orléans) ; des relations économiques et commerciales étroites avec la proximité de l'influente ville d'Orléans ; enfin, Saran profite d'un réseau de communication terrestre et fluvial dense mis en place dès la très haute Antiquité, qui permet d'assurer la diffusion de ses productions. Trois secteurs de productions ont été reconnus au cours des opérations archéologiques menées depuis les premières découvertes [ill. 1]. La zone de production dite du « Bourg sud » est composée de plusieurs ateliers où sont attestés 39 fours de potiers/tuiliers répartis sur une dizaine d'hectares. Il s'agit à ce jour de la plus grande zone potière connue pour le haut Moyen Âge dans le nord de l'Europe.

\section{Une argile utilisée sans préparation notable}

Grâce aux observations macroscopiques et aux analyses physico-chimiques, il est possible d'étudier les caractéristiques des matières premières argileuses utilisées pour le façonnage des céramiques, leur transformation et leur évolution au cours du temps et d'aborder la question de la diffusion des produits. Les céramiques produites au lieu-dit la Guignace à Saran (Bouillon, Capron, 2015), appartenant à plusieurs contextes datés $\mathrm{du} \mathrm{V}^{\mathrm{e}} \mathrm{au} \mathrm{IX}^{\mathrm{e}}$ siècle, sont caractérisées par l'utilisation d'une matière argileuse relativement homogène et riche en inclusions siliceuses. Cependant, une certaine hétérogénéité des pâtes est observable à la loupe et par les analyses chimiques ${ }^{\mathbf{1}}$. Ces premières analyses témoignent de l'utilisation d'un matériau argileux où la smectite domine avec des pourcentages élevés en oxydes d'aluminium (entre 13 et $21 \%$ ), de silicium (entre 70 et $78 \%$ ), de potassium (entre 1,5 et 2,5\%) et de fer (entre 2,9 et $5,5 \%$ ). Le silicium est lié non seulement à la composition de l'argile, mais également aux inclusions siliceuses qui constituent l'essentiel du cortège minéralogique des éléments non plastiques des céramiques. Les principales différences entre les pâtes reposent sur les variations des teneurs en alcalins et alcalino-terreux, en titane et des éléments en faible proportion comme le baryum et le zirconium. La texture de la pâte et sa couleur sont également des paramètres pris en compte pour mettre en évidence des modifications dans le processus de la chaîne opératoire (composition du matériau ou changement dans sa préparation ou sa cuisson).

Ces variations de composition et de texture n'ont pas pu être associées à un type d'objet (vaisselle de table, vaisselle de préparation ou vaisselle culinaire par exemple). En revanche, certains groupes semblent pouvoir être liés à un mode de cuisson distinct, peut-être associable à une évolution chronologique. Les céramiques classées dans le groupe 1 sont caractérisées non seulement par une composition chimique différente, mais aussi par une pâte au cœur plutôt beige-orangé alors que la couleur de surface des vases est généralement sombre, caractéristique d'une fin de cuisson en atmosphère réductrice [ill. 2]. Les pâtes de ces céramiques attribuées à la phase $8 \mathrm{~A}\left(\mathrm{VIII}^{\mathrm{e}}\right.$ siècle $)$ présentent également en abondance des inclusions hétérogranulaires $(\geq 0,5 \mathrm{~mm})$ non plastiques. Les groupes 2 et 3 intègrent les productions les plus précoces de l'atelier de Saran ( $\mathrm{V}^{\mathrm{e}}-\mathrm{VII}^{\mathrm{e}}$ siècle). Ils présentent une surface claire, blanchâtre à beige. Les céramiques classées dans ces deux groupes se distinguent non seulement par la couleur de la pâte (beige-orangé ou grise) et la granulométrie plus fine des inclusions, mais aussi par des taux plus élevés en aluminium dans l'un des deux groupes. À travers la discrimination de ceux-ci, on perçoit des approvisionnements en matières premières, éventuellement des préparations du matériau comme son épuration ${ }^{2}$ et des conditions de cuisson variées. Enfin, le groupe 4 rassemble des productions du $\mathrm{IX}^{\mathrm{e}}$ siècle ; la surface de ces céramiques est plutôt de couleur grise, la couleur de la pâte dans la tranche varie de l'orange au gris et les teneurs en oxyde de silicium sont très élevées (77\% en moyenne).

L'altération de quelques échantillons interdit de les classer dans les groupes précédents. Elle se manifeste par une augmentation des proportions de calcium et baryum. De la barytine (sulfate de baryum) a par ailleurs été identifiée au microscope électronique à balayage dans un fond de céramique provenant de la ZAC des Vergers ( $\mathrm{VII}^{\mathrm{e}}$-VIII ${ }^{\mathrm{e}}$ siècle) (Dessandier, Jézéquel, 2001, p. 7 ; Pauly, 2008). D'autres analyses physico-chimiques ont été réalisées sur des échantillons provenant de la Médecinerie ( $\mathrm{VI}^{\mathrm{e}}-\mathrm{VII}^{\mathrm{e}}$ siècle) sur un nombre restreint d'échantillons (argiles crues et tegulae). Elles mettent en évidence des similitudes entre les matières premières et les terres cuites prélevées à la Guignace, à la Médecinerie et à la ZAC des Vergers. Il est cependant difficile d'exploiter plus avant des résultats obtenus par des techniques d'analyses différentes et de spécifier les degrés de similarité des approvisionnements en matière première ou encore le type de préparation de celle-ci. L'ensemble des analyses suggère cependant que les potiers de Saran ont utilisé les ressources argileuses issues des formations argilo-limoneuses du plio-miocène, en privilégiant la formation des sables et argiles de Sologne qui affleurent localement et qui se distinguent par l'absence de carbonates, contrairement au niveau supérieur constitué des sables, argiles et marnes de l'Orléanais. Ces matériaux présentent de 


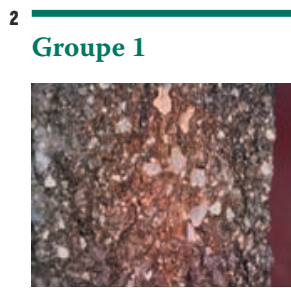

1890-5 La Guignace

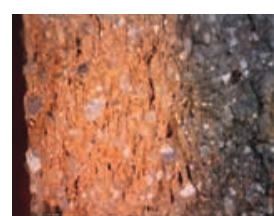

1978-4 La Guignace

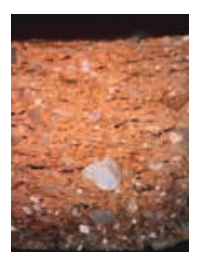

1668-1

La Guignace

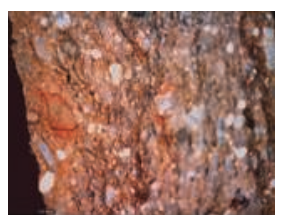

1556-1 La Guignace

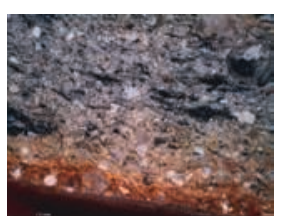

1573-2 La Guignace

Groupe 2

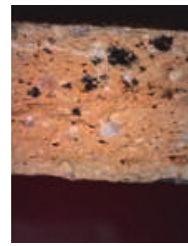

3024-12

La Guignace

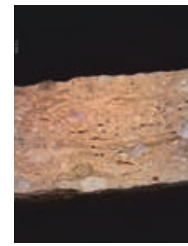

2731-1

La Guignace

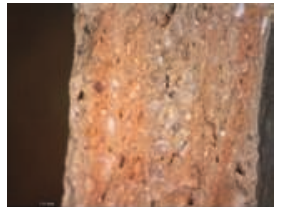

2012-1 La Guignace

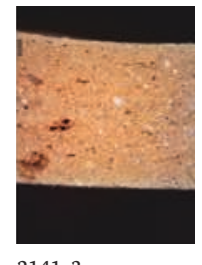

2141-3

La Guignace

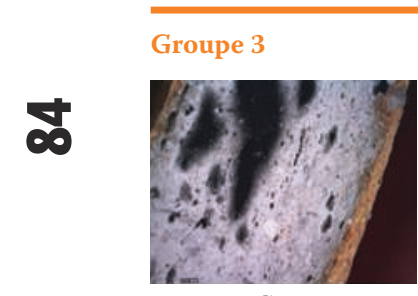

2106-1 La Guignace

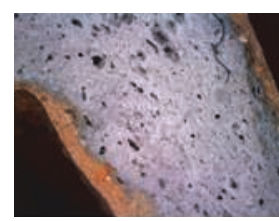

2141-7 La Guignace

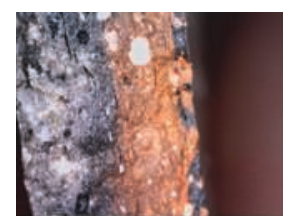

3023-1 La Guignace

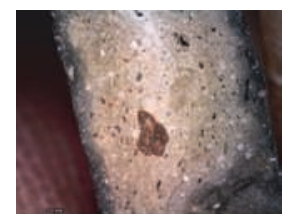

36a-La Médecinerie

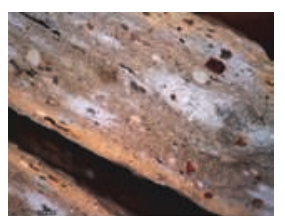

3027-2 La Guignace

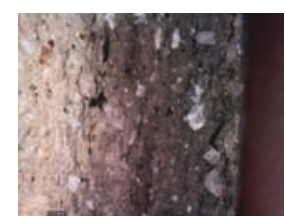

2012-2 La Guignace

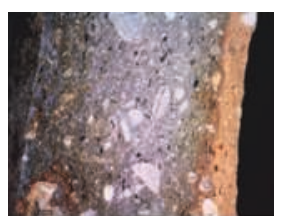

2731-7 La Guignace
Groupe 4

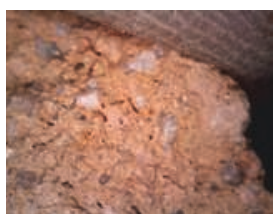

1062-2 La Guignace

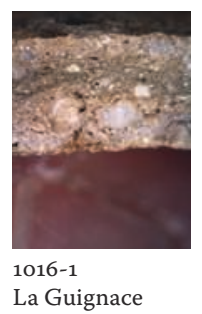

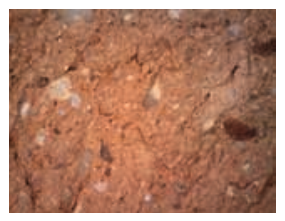

1890-8 La Guignace

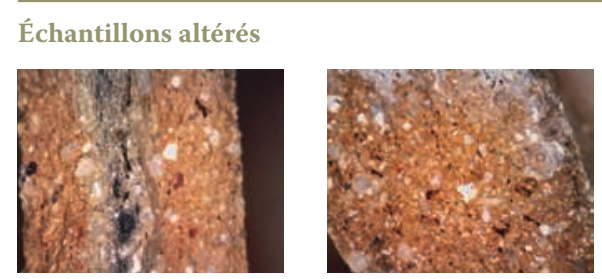

2791-1 La Guignace
1668-4. La Guignace

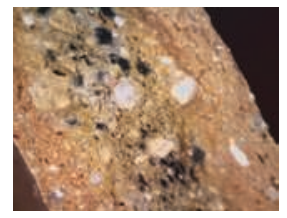

2731-4 La Guignace

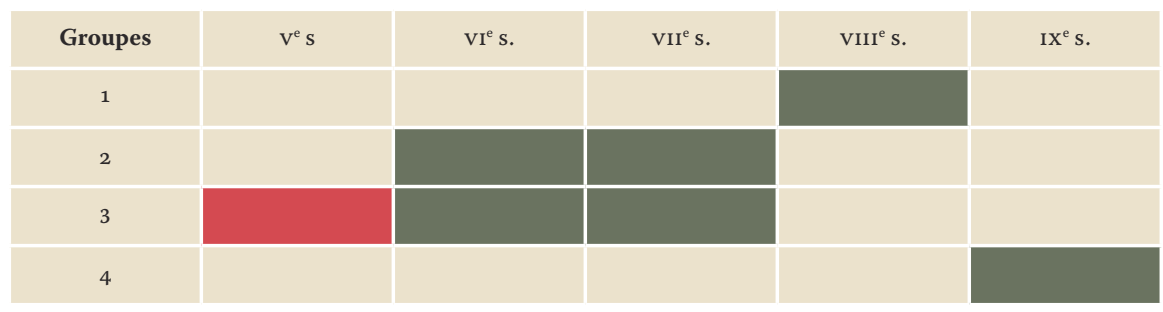


2. Les quatre groupes

macroscopiques des

échantillons prélevés

parmi le corpus céramique

du site de la Guignace

et de la Médecinerie

3. Vue partielle de la grande

carrière d'argile découverte

à la Guignace. La formation

des sables et argiles

de Sologne de couleur jaune

orangé, visible au fond

du sondage au premier plan,

a été exploitée sur une

profondeur de l'ordre

de $1,40 \mathrm{~m}$ représentant

a minima $1206 \mathrm{~m}^{3}$ d'argile

prélevée.
3. Dans la coupe sud

du lac de la Médecinerie, entre les fours D et C.

4. Une fosse

quadrangulaire comblée

d'argile, retrouvée dans

la zone d'inhumation

attenante à l'atelier

de la ZAC des Vergers,

pourrait correspondre

à une tombe réutilisée.

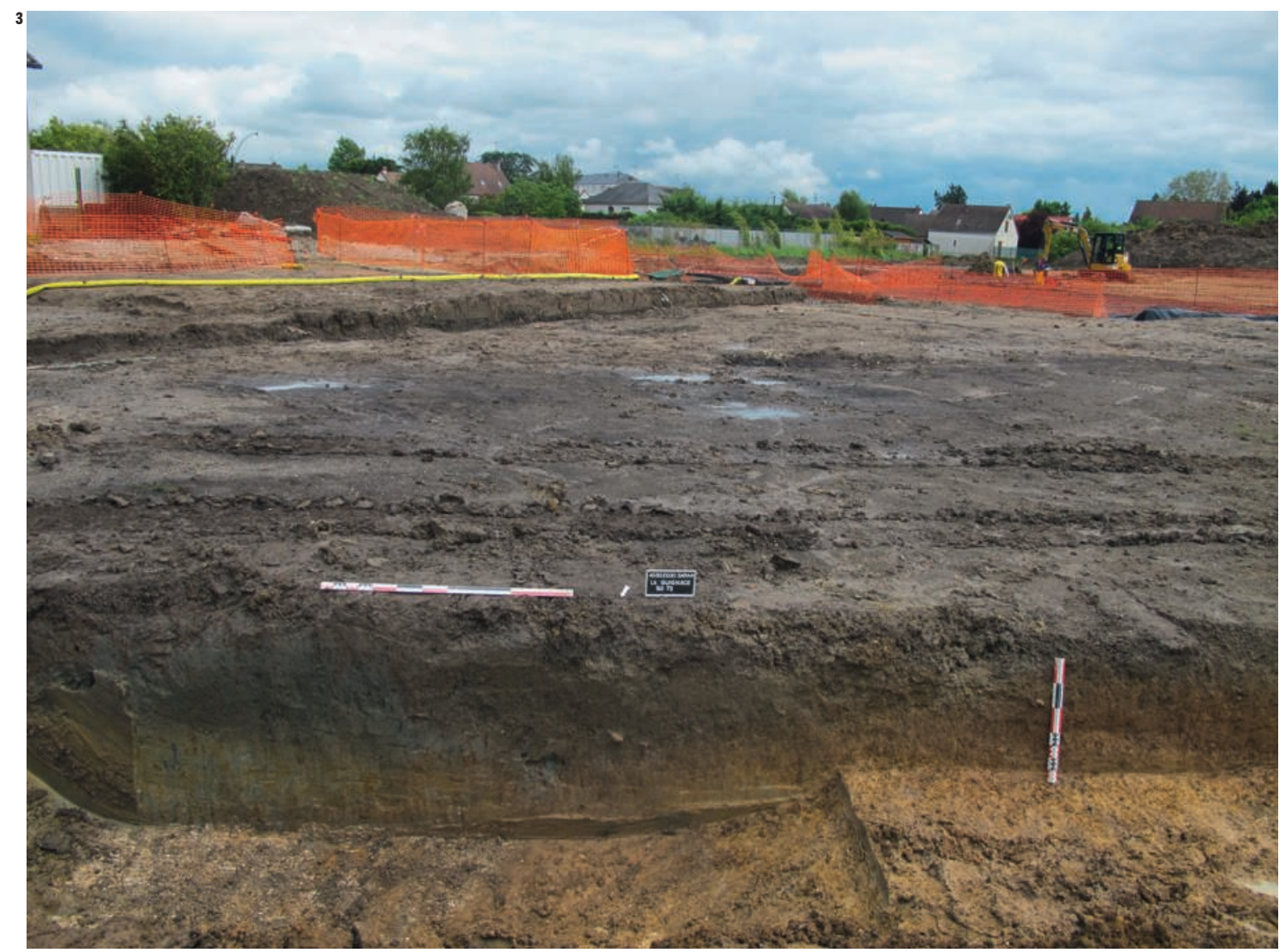

nombreuses variations de faciès, notamment granulométriques (Berger, Desprez, 1969). La limite entre les deux formations est difficile à appréhender, ce qui explique probablement l'utilisation par les potiers de matériaux aux caractéristiques distinctes.

L'exploitation de la matière argileuse dans les niveaux géologiques superficiels a pu être attestée en différentes zones de l'aire de production. Deux formes d'extraction ont pu être appréhendées : l'une par prélèvement ponctuel superficiel, illustrée par des fosses d'envergure restreinte généralement de l'ordre du mètre carré mais pouvant atteindre quelques dizaines de mètres carrés sur une profondeur de quelques dizaines de centimètres (zone de la ZAC des Vergers) (Jesset, 2001) ; l'autre sous la forme d'une extraction de type carrière à ciel ouvert (argilière), correspondant à une vaste dépression (zone de la Guignace) (Bouillon, Capron, 2015, p. 210-211). Cette dernière, partiellement mise au jour sur une surface au sol d'environ $1855 \mathrm{~m}^{2}$, profite d'une implantation inscrite au cœur d'un talweg permettant d'établir un front de taille naturel [ill. 3].

Sur l'ensemble des zones de production, les vestiges ou indices d'activités en lien avec une quelconque préparation de l'argile sont rares, voire inexistants. À en rester à cette échelle d'analyse, une utilisation en « terre franche », c'est-à-dire sans traitement particulier de la matière première
(Guadagnin, 2000, p. 55), paraît envisageable, certaines zones offrant d'ailleurs une argile pure ne nécessitant a priori aucun traitement de décantation ou de tamisage. Toutefois, les résultats des études pétrographiques semblent indiquer dans certains cas l'ajout de dégraissants, qui nécessiterait un mélange de l'argile et du sable, probablement par foulage (Jesset, 2001). Cette activité n'a pas laissé de trace tangible, à l'exception d'une fosse de pourrissage observée en 1969 par Jean Chapelot $^{3}$ : si elle s'avère, à la lumière de nouvelles explorations, être le prolongement de la voie antique qui traverse l'atelier (Chapelot, 1969), on ne peut exclure l'utilisation du revêtement de la voie pour le foulage ou le marchage de l'argile.

Les structures de stockage de l'argile sont tout aussi rares, découvertes dans l'environnement immédiat des fours au sein de l'atelier, sous la forme de quelques fosses de plan rectangulaire ou circulaire, sans aménagement particulier, et comblées d'une argile plastique. L'argile stockée dans ces structures rudimentaires, parfois réutilisées de façon opportuniste ${ }^{\mathbf{4}}$ (Jesset, 2001, p. 128), pourrait aussi bien avoir servi à la construction ou à la réfection des fours qu'au montage des céramiques.

\section{Les traces du tournage}

Aux marques de tournage telles que les stries et cannelures, résultat du façonnage manuel pratiqué 


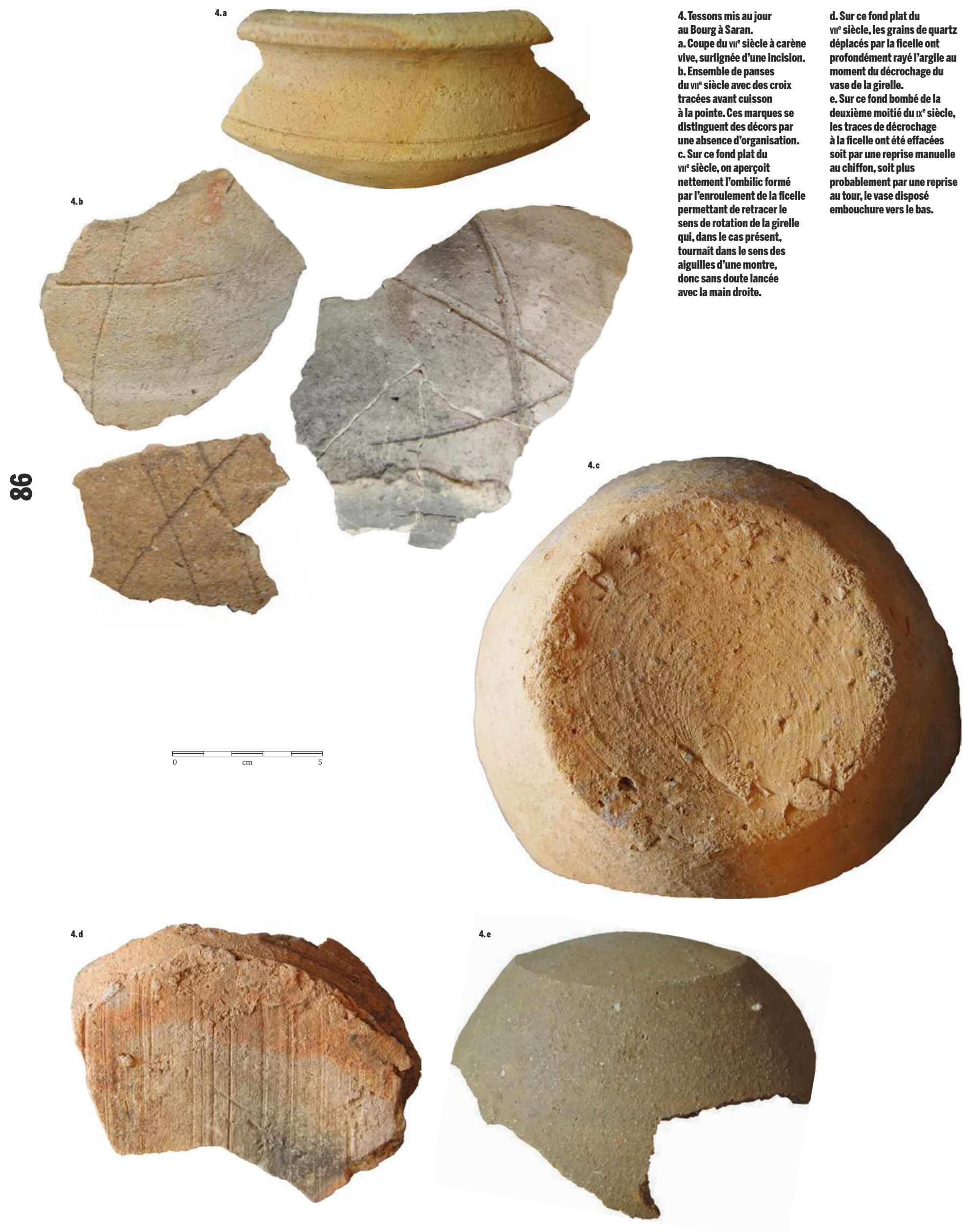


par le potier, s'ajoutent les stigmates involontaires et ponctuels. Les rayures (dégraissants de surface traînés lors du tournage), les enfoncements plus ou moins marqués ou les digitations légères témoignent de manipulations. Elles interviennent probablement lors de l'enlèvement du vase de la girelle pour le transporter vers l'aire de séchage ou au cours du séchage. Certaines traces se rapportent à des gestes précis opérés au cours du tournage. C'est le cas des ressauts, carènes ou incisions horizontales réalisés au moyen d'un objet pointu ou d'une estèque [ill. 4a]. Ces traces cloisonnent la surface du vase en différents registres qui servent à l'application d'un décor ou surlignent des ruptures dans le profil du vase. Quelques marques anépigraphes font également penser à des marques de tâcherons, comme les croix tracées rapidement à la pointe du couteau sur les panses des pots et retrouvées en nombre important dans le comblement du four du site du Bourg [ill. 4b]. Ce type de marquage peut être rapproché de celui identifié sur les tegulae.

La base des céramiques présente régulièrement les marques du décrochage réalisé généralement à l'aide d'un fil. Ainsi, les traces sont fréquemment concentriques sur les récipients de petit à moyen module [ill. 4c], signe d'un tour encore en action lors du décrochage. Elles apparaissent linéaires sur les individus de plus grand gabarit, indice d'un retrait à la ficelle se faisant tour à l'arrêt [ill. 4d]. L'évolution morphologique de la base des vases au cours des siècles éclaire celle des techniques de montage. Par exemple, le profil lenticulaire des fonds identifié sur les pots et cruches à partir du $\mathrm{VIII}^{\mathrm{e}}$ siècle témoigne d'un type de façonnage en rupture avec les fonds plats qui étaient en usage jusqu'alors. Ainsi, les fonds lenticulaires présentent sur leur partie interne de nombreuses traces de mise en forme effectuées sans doute hors du tour et une reprise de l'extérieur du fond qui a fait disparaître toutes traces d'enlèvement de la girelle [ill. 4e]. Cette opération de façonnage manuel semble liée à la volonté d'amincir les parois, solution aux problèmes récurrents de séchage, rencontrés préalablement sur les fonds plus épais et qui généraient de multiples éclatements au cours de la cuisson. Cette étape de reprise hors du tour doit sans doute affecter les cadences de tournage.

Sur les différentes zones de production de Saran, cinq ou six fosses de tour à axe fixe sont attestées [ill. 5]. Parallèlement, la mise au jour de crapaudines en pierre dure, sans doute intégrées au système de rotation de l'axe ou de la girelle, confirme l'existence de ces structures discrètes [ill. 6]. La phase de tournage n'est cependant pas systématique, certaines formes plus petites témoignant d'une mise en forme par modelage [ill. 7]. Le façonnage manuel concerne également les ajouts des systèmes de préhension ou les éléments verseurs (anse, oreille, bec pincé ou tréflé) et les percements (oreille de suspension, couvercle) opérés tour arrêté. Cette étape intervient dans tous les cas après la décoration du vase, comme le montrent les nombreux exemples de recouvrement des décors par l'accroche des anses ou systèmes de préhension [ill. 8a].

Des ajouts de matière sont parfois identifiés sur certains vases. Ainsi, au IX ${ }^{\mathrm{e}}$ siècle, l'apparition de formes de grands gabarits (mortiers ou grandes jattes) entraîne un épaississement des parois nécessitant un renforcement sous la forme de bandes d'argile généralement verticales. D’abord non digités, ces ajouts seront à partir de la seconde moitié du $\mathrm{IX}^{\mathrm{e}}$ siècle imprimés au doigt pour pallier le manque de cohésion ou d'adhérence de la pâte après collage à la barbotine. Ce procédé trouve ses limites sur l'atelier de Saran si l'on en juge par les fréquentes traces de décollage de ces parties ajoutées.

\section{L'évolution des décors}

L'atelier offre sur ses cinq siècles d'activité des productions pour lesquelles le traitement de surface constitue un caractère technologique discriminant. Les céramiques sans revêtement réunissent les pâtes brutes, mais aussi celles subissant un polissage partiel ou total occasionnant un facettage des surfaces (aspect résultant d'une orientation changeante du sens de frottement). Ce type de traitement de la surface s'opère au stade cuir de sa consistance avec un objet dur (os, pierre, bois dur...), dont les vestiges archéologiques font défaut. Les productions les plus précoces exposent régulièrement une surface enfumée associée parfois à un polissage [ill. 8b]. Le principal traitement de surface observé consiste en un revêtement de type engobe. Les exemplaires mis au jour illustrent une application par trempage ou au pinceau [ill. 8c]. Cette technique, souvent combinée à un polissage, est en plein essor au milieu du VIII ${ }^{\mathrm{e}}$ siècle. Elle a également été récemment mise en évidence, quoique dans des proportions marginales, sur des productions de la fin $\mathrm{du} \mathrm{VII}^{\mathrm{e}}$ siècle, voire dès le $\mathrm{VI}^{\mathrm{e}}$ siècle pour quelques tessons en attente d'analyse (Bouillon, Capron, 2015, t. II, p. 98). Ce traitement reste globalement mal maîtrisé par les potiers saranais et de fréquents écaillages des surfaces sont perçus, identifiés aussi sur les exemplaires en contexte de consommation. Ce constat suppose un réel problème général lié à cette production spécifique (séchage, cuisson, dilatation différentielle...) (Jesset, 2007). Parmi les productions les plus tardives de la zone de production de Saran (ZAC Portes du Loiret/le Mesnil), une couverte à la glaçure est parfois observée sur des vases issus de contextes du milieu $\mathrm{x}^{\mathrm{e}}$-milieu $\mathrm{XI}^{\mathrm{e}}$ siècle [ill. 8a] (Laurent-Dehecq et al., à paraître). L'utilisation de ce type de revêtement sur les productions de Saran reste cependant exceptionnelle, et le recouvrement en règle générale très partiel paraît d'origine accidentelle.

Parmi les décors manuels, les digitations sont omniprésentes. On les retrouve au VIII ${ }^{\mathrm{e}}$ siècle sur 


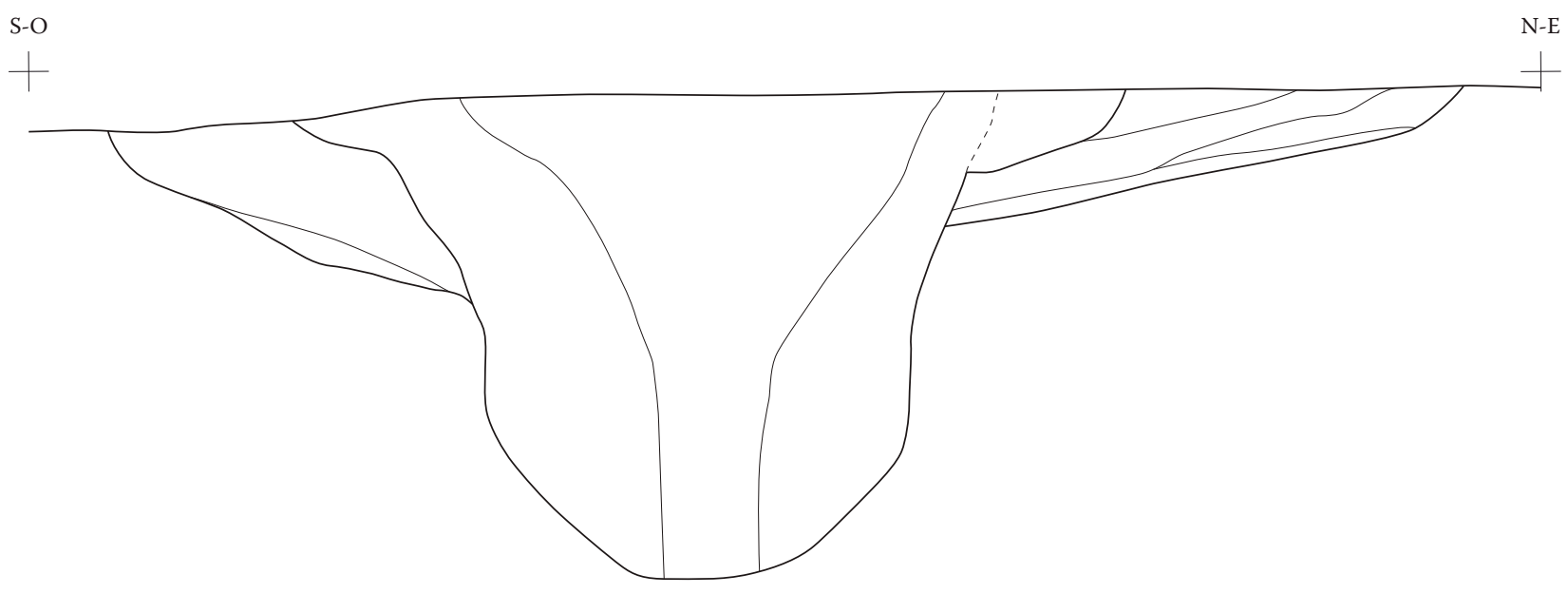

site de « la Médecinerie »

6 Exemplaire du site de Saran, « la Guignace »

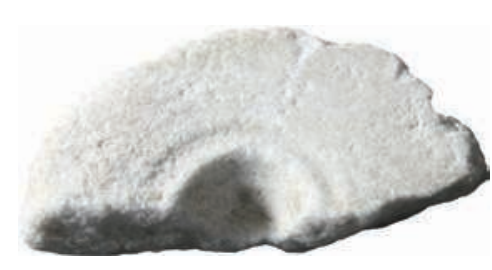

Exemplaire du site de Saran, «ZAC Portes du Loiret »

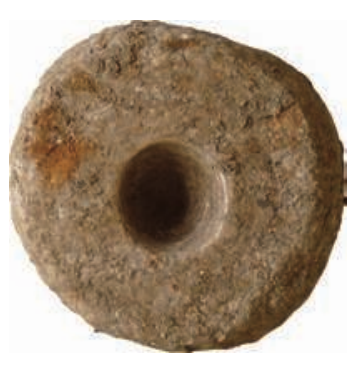

Exemplaire du site de Saran, « la Source Saint-Martin »

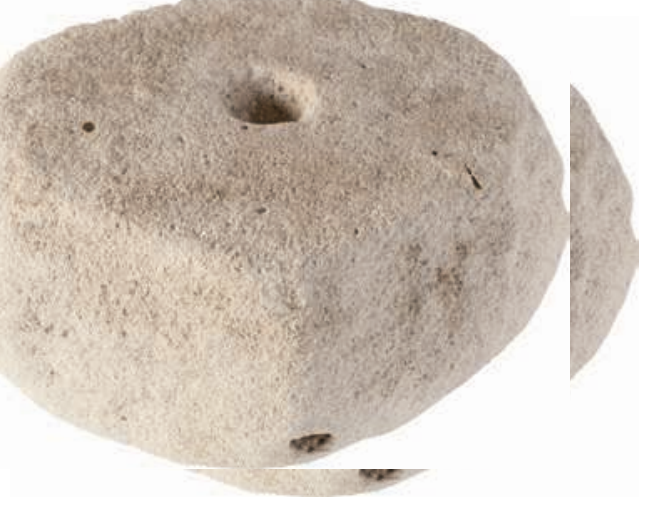


6. Les trois crapaudines

découvertes dans des

contextes de la fin du vie siècle

à la première moitié du

Ix siècle à Saran sont très

proches typologiquement.

Plates ou de forme

hémisphérique, elles sont

façonnées dans un calcaire

dur, présentent une face

supérieure plane percée

d'une loge d'usure

occasionnée par l'aiguille

et une face externe brute

qui devait se loger dans l'axe ou le volant-girelle.

7. Exemples de petites formes

simples modelées, dont

I'utilisation comme creuset

reste hypothétique (le Bourg).

8. a. Cruche à bec pincé et

anse plate large à deux gorges

opposées de la fin du $x^{e}$ siècle

(ZAC Portes du Loiret).

Elle est décorée en premier

lieu d'un registre de molette

à motifs de losanges,

puis de motifs repoussés/

pincés ayant occasionné une

déformation de la molette.

Enfin, l'anse a été fixée

sur la lèvre et en partie basse

sur l'extremum de la panse.

La coulure de glaçure

au plomb est sans doute

accidentelle.

b. Ensemble de céramiques

de la fin du ve siècle, cuites

en mode réducteur, avec

polissage des surfaces

et décor de guillochis réalisé

à l'aide d'une lame vibrante

(la Médecinerie). On remarque

la décoration au guillochis

intervenant après la phase

de polissage sur consistance

cuir et par conséquent

le faible relief du décor.

c. Col de cruche à bec

tubulaire tréflé tangent

à la lèvre de la deuxième

moitié du Ixe siècle

(la Médecinerie). L'apposition

de l'engobe sur cet exemplaire

a été faite au pinceau si l'on

en croit le déficit de couleur

(plages beige clair) visible

au revers du système verseur
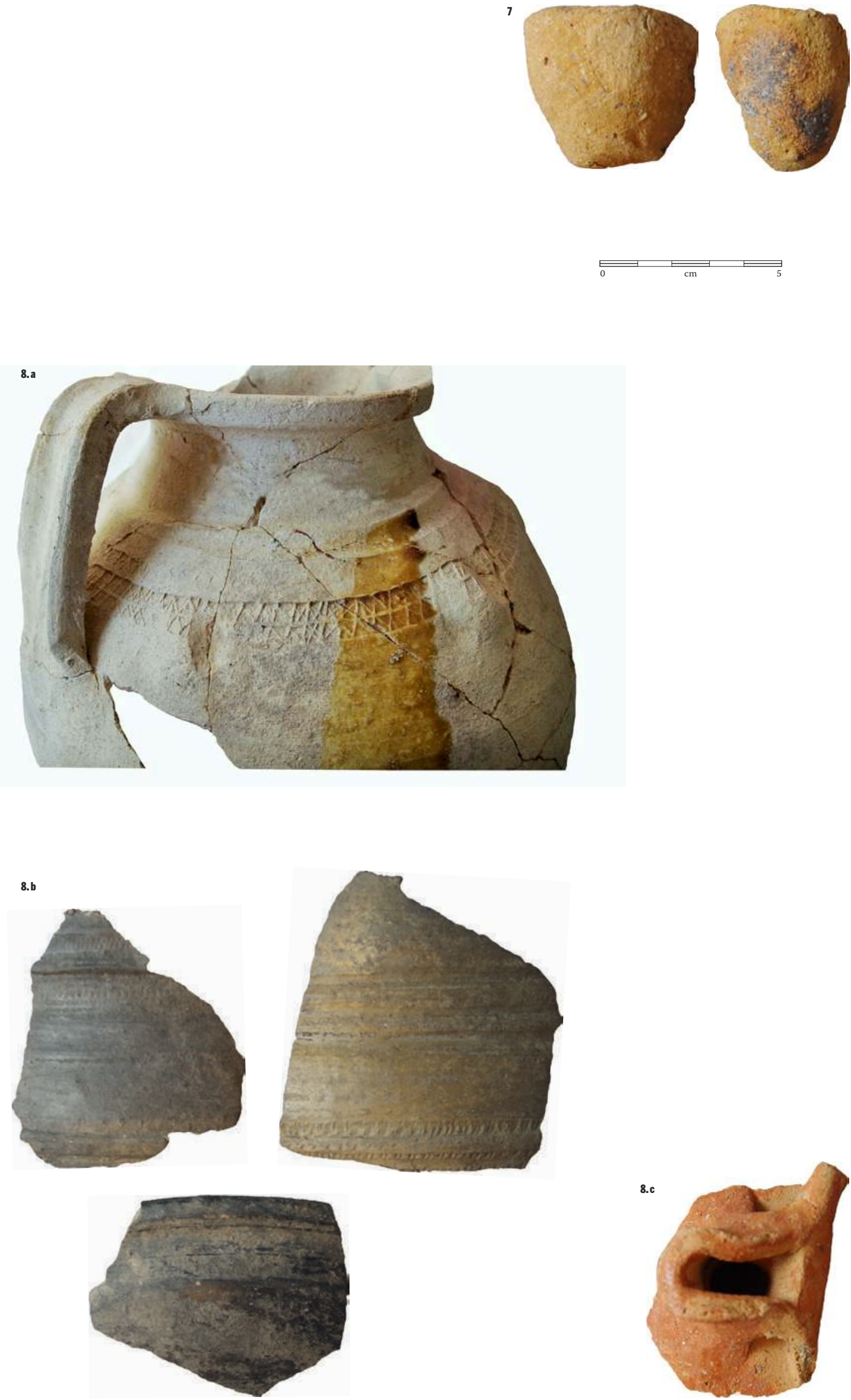

et à l'intérieur de celui-ci. 

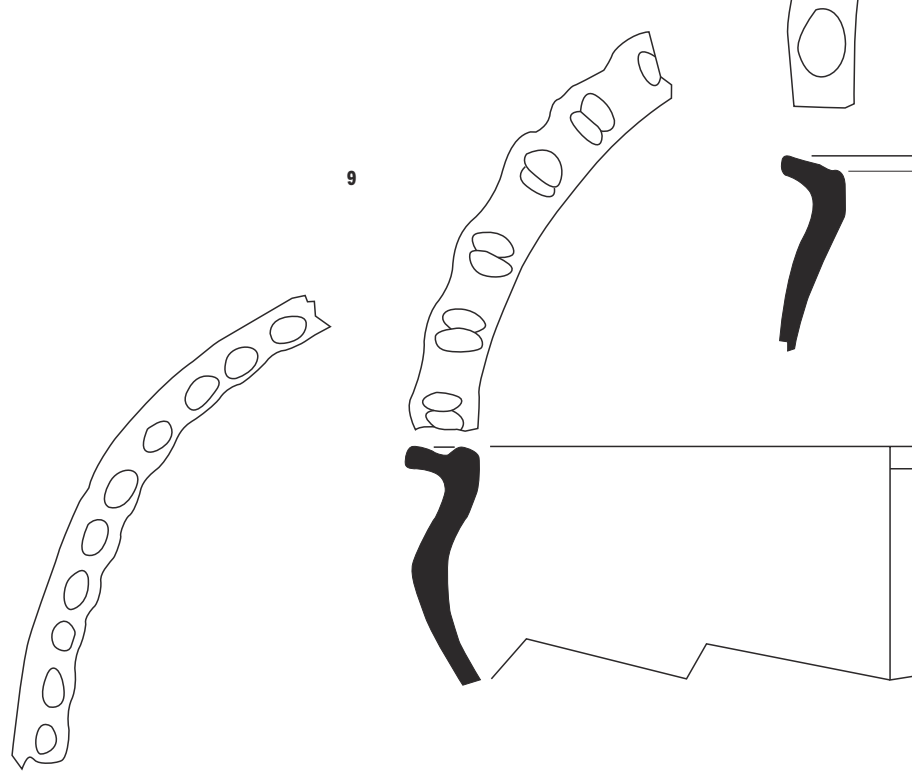

9

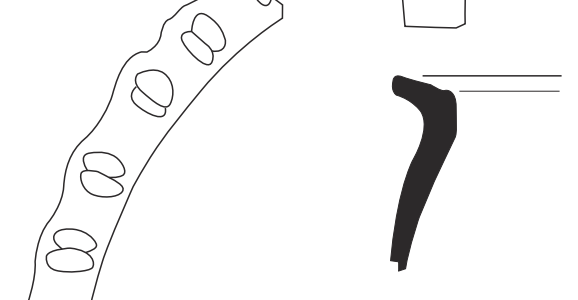

8
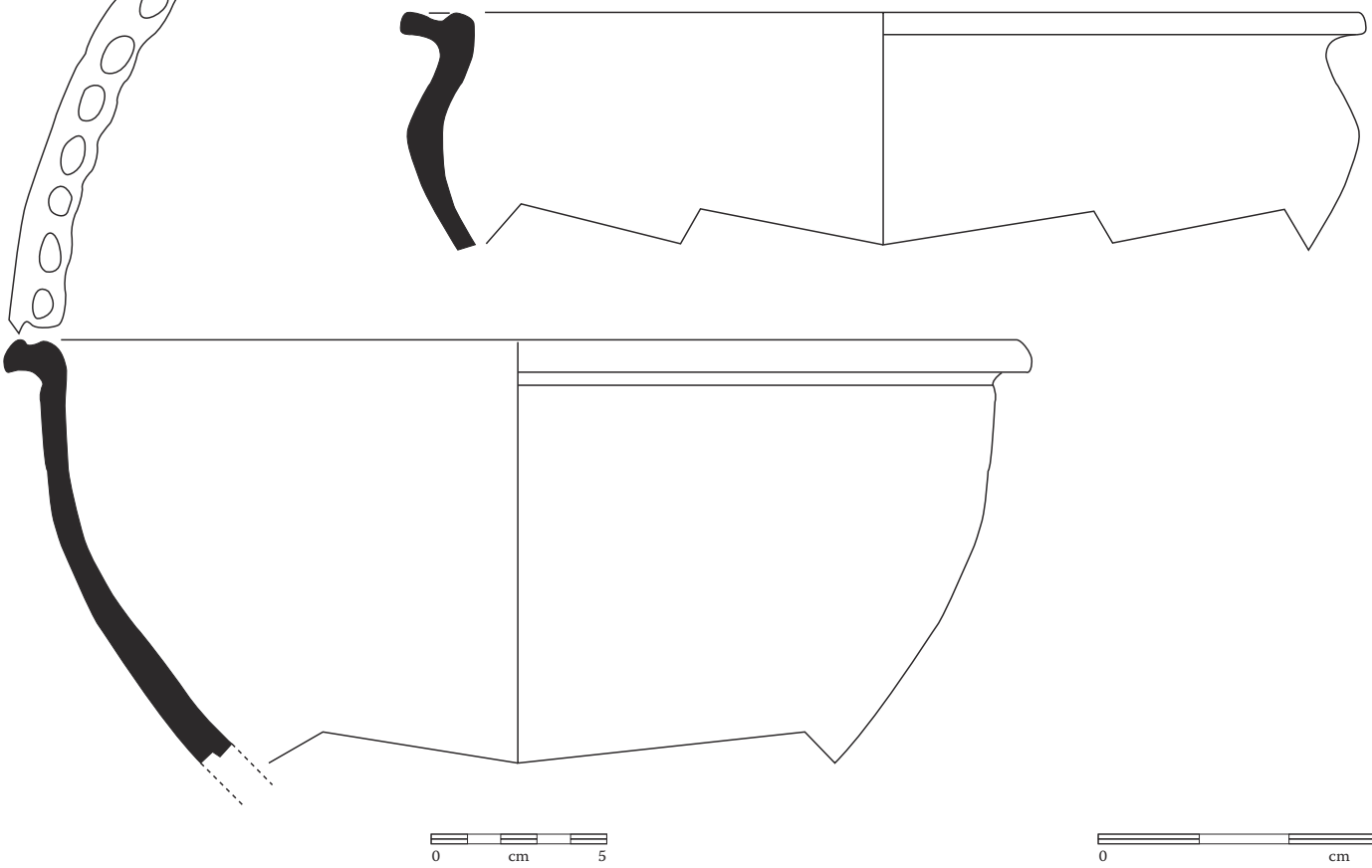

9. Les décors de digitations

observés dans de rares

cas sur les lèvres de jattes

du vilI siècle suggèrent une

pratique décorative qui reste

marginale au sein des

productions de Saran et

forme dans tous les cas un

groupe atypique pour le haut

Moyen Âge (ZAC des Vergers).

10. Gobelet de la fin du

VII -début du viII' siècle décoré

de deux registres de poinçons

de palmettes et marguerites

(Selliers à Ingré).

10

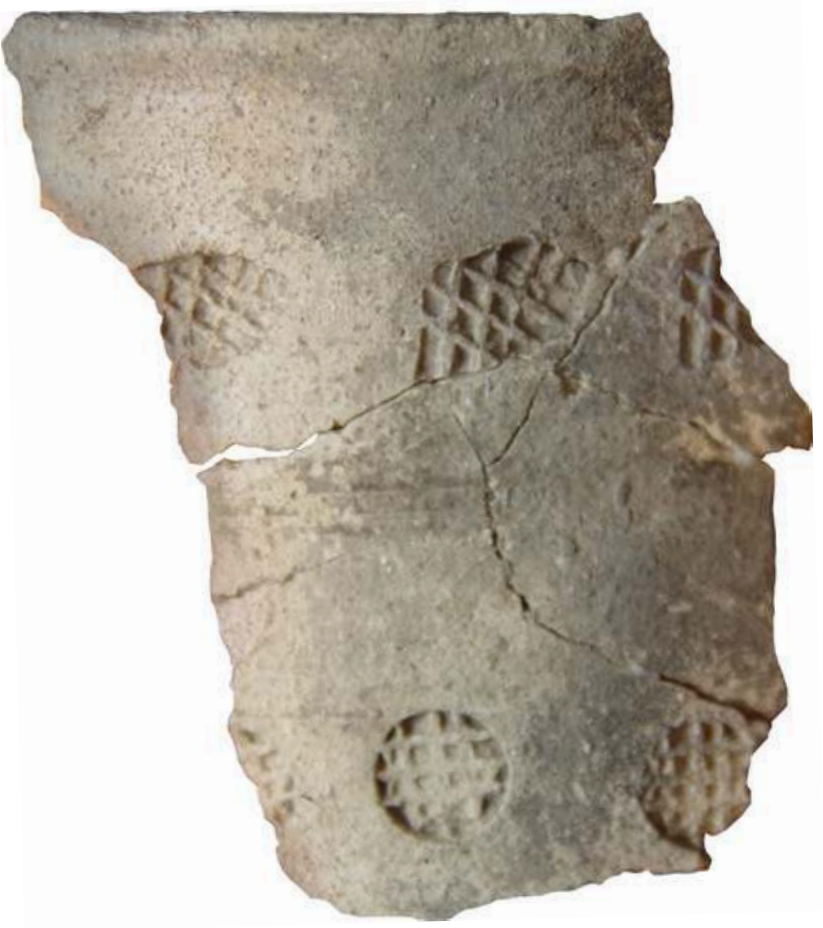


le sommet de la lèvre de certaines jattes où elles forment un cordon régulier (digitations ou pincements) [ill. 9]. En fonction des contextes appréhendés, elles sont parfois combinées à des apports de matière ; il est cependant délicat dans ces derniers cas de s'assurer de leur rôle décoratif. Dans le même temps, vers le milieu du $\mathrm{IX}^{\mathrm{e}}$ siècle, apparaissent des décors de motifs repoussés (cercles, pastilles bandes verticales ou horizontales), généralement combinés à un engobe et un polissage. Il s'agit de déformations au doigt obtenues depuis l'intérieur de la paroi du vase.

Les décors mécaniques - réalisés à l'aide d'outil (Jesset, 2013, p. 214) - sont apposés sur tous types de vases indistinctement. Les artisans saranais utilisent principalement le décor à la molette, généralement apposé sur un ou deux registres au moyen d'une roulette en bois gravée de motifs simples et dont aucun vestige ne nous est parvenu à ce jour. Le module du cylindre des roulettes se réduit au cours du temps : compris entre $1,5 \mathrm{~cm}$ et $2,5 \mathrm{~cm}$ à la période mérovingienne, il est inférieur à 1,5 cm à la période carolingienne. Même si une étape de séchage semble nécessaire avant l'apposition de la roulette, il apparaît à Saran que ce décor s'effectue lors du tournage. L'usure des motifs sur certains rebuts de cuisson atteste d'une utilisation répétée de la roulette dont la fragilité peut être perçue par sa dégradation progressive et notamment la perte de détails, voire de motifs complets, lors de l'impression. L'application de la molette se fait sans doute sur le tour, ce qui explique le recouvrement souvent observé entre le début et la fin du développé du décor. Sur certains vases, la présence d'un registre très faiblement imprimé et légèrement décalé par rapport au motif final pourrait indiquer une première rotation de réglage afin de tester la position du décor. Cette pratique, qui ne paraît pas généralisée, fournit toutefois un éclairage sur l'attention relative portée par certains potiers à ce type de décoration.

L'empâtement ou le faible relief des molettes visibles sur certaines pièces montre à l'inverse une application ne respectant pas les temps de séchage optimum. D’autres décors mécaniques plus anecdotiques sont appliqués par les artisans. Il s'agit d'incisions ondées, entrecroisées ou non, réalisées à la pointe, de poinçons, dont les exemplaires, limités aux $\mathrm{V}^{\mathrm{e}}$-V $\mathrm{v}^{\mathrm{e}}$ siècles, restent très rares à Saran [ill. 10], et de guillochis, décor utilisé à la même période. Sur les éléments lustrés, ce dernier type de décor intervient dans un second temps.

\section{La transformation des fours au VIII ${ }^{\mathrm{e}}$ siècle}

La teinte des céramiques au cours des différentes phases de production connaît des changements [encadré]. On passe vers le milieu du VIII ${ }^{\mathrm{e}}$ siècle de céramiques bien cuites, à sonorité métallique, aux parois de 6 à $8 \mathrm{~mm}$ d'épaisseur, avec une teinte jaune à orangée et un cœur gris ardoisé, en place depuis le $\mathrm{vI}^{\mathrm{e}}$ siècle, à des productions brun-gris à beige de 2 à $4 \mathrm{~mm}$ d'épaisseur, avec des surfaces plus granuleuses et une sonorité plus mate, conséquence d'une cuisson moins intense. Ce constat se couple avec celui fait sur l'évolution architecturale des fours.

Les plus précoces, de plan quadrangulaire et à foyer ouvert, présentent un fond plan et une sole surélevée au moyen de pilettes maçonnées. La hauteur de ce type de four n'excède pas $1 \mathrm{~m}$ entre la sole et le haut de la chambre de cuisson [ill. 11]. À partir de la seconde moitié du VIII ${ }^{\mathrm{e}}$ siècle, la chambre devient exclusivement circulaire. On observe alors une pente ascendante comprise entre 5 et $15 \%$, depuis l'ouverture de l'alandier jusqu'à la chambre de cuisson. Celle-ci est aménagée dans le meilleur des cas d'une languette maçonnée ou réservée dans le terrain naturel.

C'est probablement ces modifications structurelles des fours, en lien avec des modes de fonctionnement différents, qui sont à l'origine de ces deux groupes céramiques clairement identifiés. Le premier four, ouvert, avec deux foyers, voit son tirage vraisemblablement insuffisant compensé par la bonne répartition de la chaleur, tandis que le second, de dimensions plus restreintes, avec une chambre de cuisson fermée et un foyer unique, nécessite des aménagements fonctionnels. Ils semblent rester insuffisants au regard de certains aspects des céramiques de cette période. En effet, l'amincissement des parois, particulièrement visible au $\mathrm{IX}^{\mathrm{e}}$ siècle, devient un phénomène récurrent. Outre la volonté de limiter les problèmes de cuisson, on peut supposer que ce façonnage répond à un souci d'économie de bois, l'affinement des parois favorisant une cuisson plus rapide des pièces, et par conséquent une consommation réduite en combustible, peut-être liée à l'évolution de la gestion forestière.

\section{Les ratés de cuisson et les problèmes de séchage}

La fouille des différentes zones de production de Saran depuis la fin des années 1960 a généré la découverte d'une grande quantité de rebuts de cuisson. Plusieurs tonnes de tessons viennent illustrer les différentes difficultés rencontrées par les artisans. Il s'agit en grande majorité de desquamations, dégradation par « plaques » plus ou moins épaisses, résultant d'un séchage imparfait en raison de surépaisseurs, combiné à une montée en température de la cuisson trop violente. Ce type de problème est principalement perceptible sur les éléments les plus massifs des vases que sont les fonds, les anses, parfois les lèvres et plus rarement les panses [ill. 12a]. Peuvent s'y associer des fissures [ill. 12b] en raison d'un séchage différentiel entraînant des rétractions ${ }^{\mathbf{5}}$ (Jesset, 2008, p. 176), des déformations [ill. 12c], des tensions, voire des déchirements de parois. Ce phénomène est souvent accentué par la présence d'inclusions grossières qui constituent des zones de faiblesses. Ces lacunes peuvent se voir renforcées lors
5. Les expérimentations de séchage conduites argileuse de Saran ont montré une rétraction au séchage de l'ordre de $10 \%$ en moyenne. 
11 Four 11
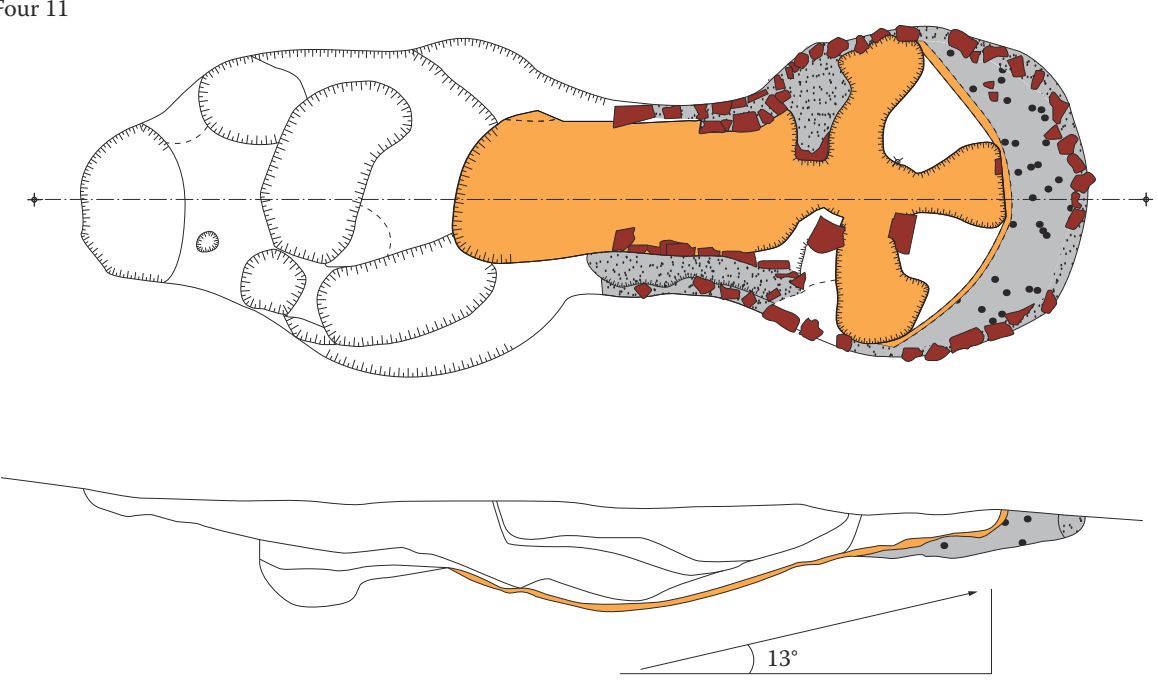

Four 8

รั

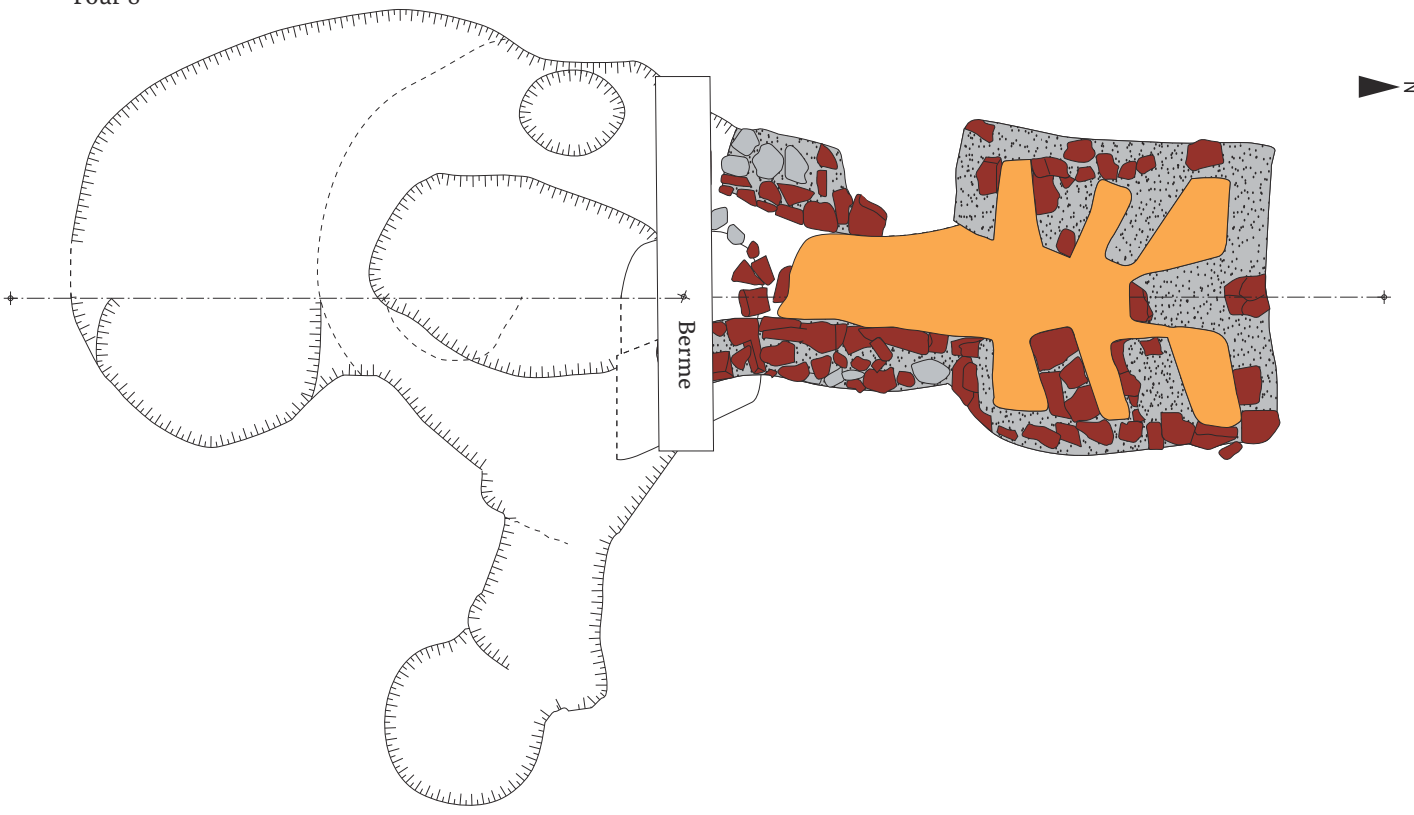

11. Plans et coupes du four 8 (seconde moitié vil ${ }^{e}$ siècle)

et du four 11 (seconde moitié

vilie siècle) du site de

la Guignace, illustrant les

mutations architecturales

des structures de cuisson.

Parmi les aménagements

innovants, une pente,

ici de $13^{\circ}$, concue pour

accélérer et intensifier

la chaleur au sein de

la chambre de cuisson.

12. Tessons de la Médecinerie

a. La surépaisseur de ce

fond de pot à cuire du

VII ${ }^{e}$ siècle a occasionné une

desquamation importante

et le rejet du vase considéré

comme rebut de cuisson.

b. Ce pot à cuire du vile siècle

présente une fissure

longitudinale depuis

l'embouchure jusqu'à un

grain de quartz proéminent

qui a sans doute favorisé

sa création.

c.Affaissement de l'épaule

d'un pot du Ix siècle, décoré

d'un motif de carrés à

la molette.ll faut sans

doute y voir un problème

de séchage.

13. Il est parfois possible,

sur quelques tessons

très particuliers, de repérer

un instant précis du travail

du potier. C'est le cas

des fragments de poteries

témoignant de la distraction

de l'artisan, d'un oubli dans

une routine pourtant bien

rodée. À ce jour, trois erreurs

ont été constatées sur

les centaines de milliers

de tessons observés:

l'absence de percement

d'un pot à oreilles, une

anse manquante au revers

d'une cruche, et le cas ici

illustré du bec tubulaire tréflé

non percé et donc inutilisable.

Si certains peuvent y voir une

sorte de " pichet trompeur ",

nous y voyons plutôt

la marque d'un façonnage

mécanique réglé en

différentes étapes, parfois

rompu par l'erreur humaine.
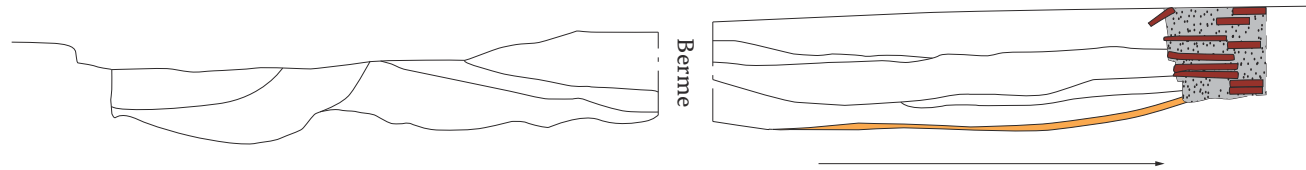

$\therefore$ mortier

๑. massif argileux

$\square$ calcaire

TCA 

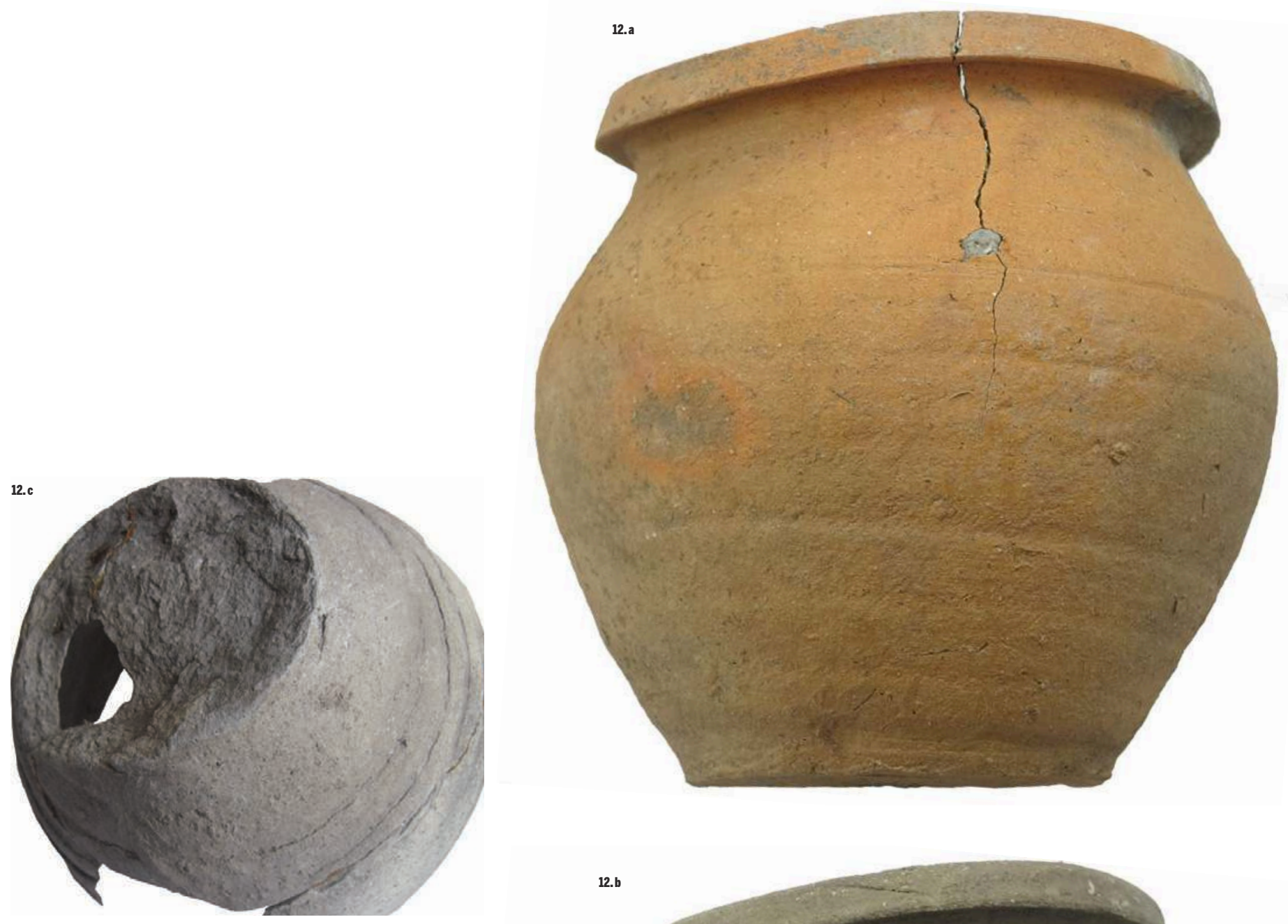

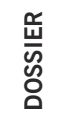

ณ

13

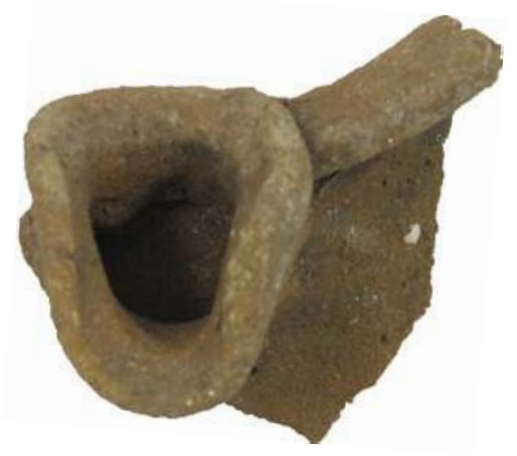

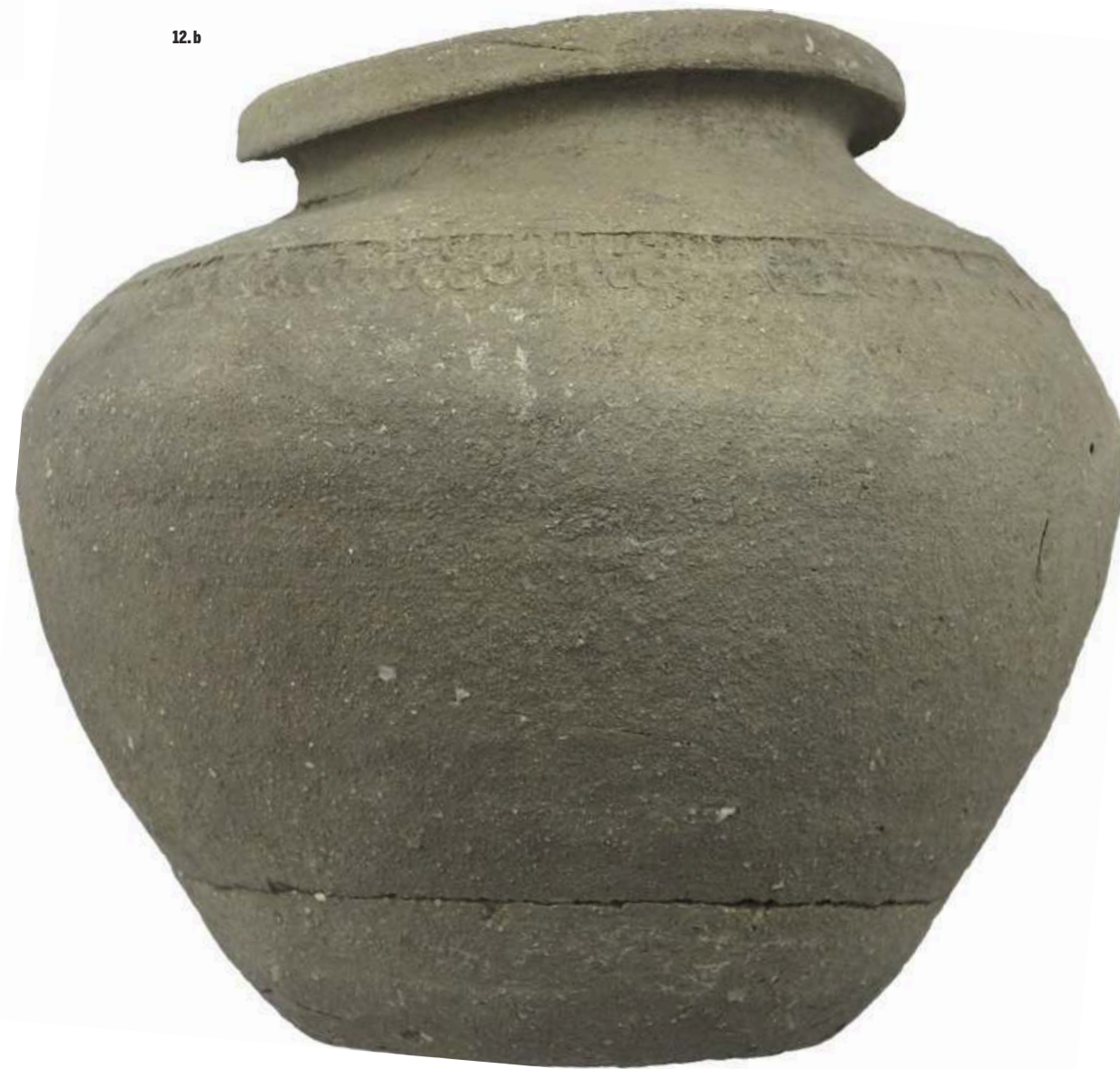

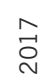

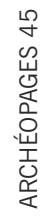


Les apports de l'expérimentation

Une grande partie des vases recueillis dans les fours des $\mathrm{vI}^{\mathrm{e}}$-vIII ${ }^{\mathrm{e}}$ siècles présentent en surface des traces gris-bleuté [ill. 1], qui tendent à s'expliquer une fois confrontées à l'expérimentation menée en 2011 sur un four à double foyer : un foyer bas alimenté classiquement au-devant de l'alandier et un foyer haut installé au sommet du laboratoire au-dessus de la charge à cuire [ill. 2] (Millet, 2015). Il est apparu au cours de la cuisson expérimentale que les surfaces à l'air libre des vases présentaient une teinte orangée bien réoxydée, tandis que les teintes grisâtres apparaissaient sur les surfaces des vases recouvertes de cendres, provenant de la combustion du foyer haut. Ces indices permettent de restituer le positionnement originel des pièces au sein du four. Ainsi, il est possible d'exclure l'enfournement de piles complètes et de privilégier au contraire une disposition relativement anarchique des pièces, embouchure vers le bas et les unes sur les autres. Ce positionnement se voit confirmé par la coloration grisâtre semi-circulaire présente sur le fond de certains vases. En outre, la superposition des pièces se révèle également par la couleur beige-verdâtre des intérieurs, occasionnée par une atmosphère confinée, sans réoxydation possible. Ces mises en œuvre se confirment aussi dans quelques cas par les empreintes de lèvres légèrement imprimées sur le fond du vase sous-jacent.

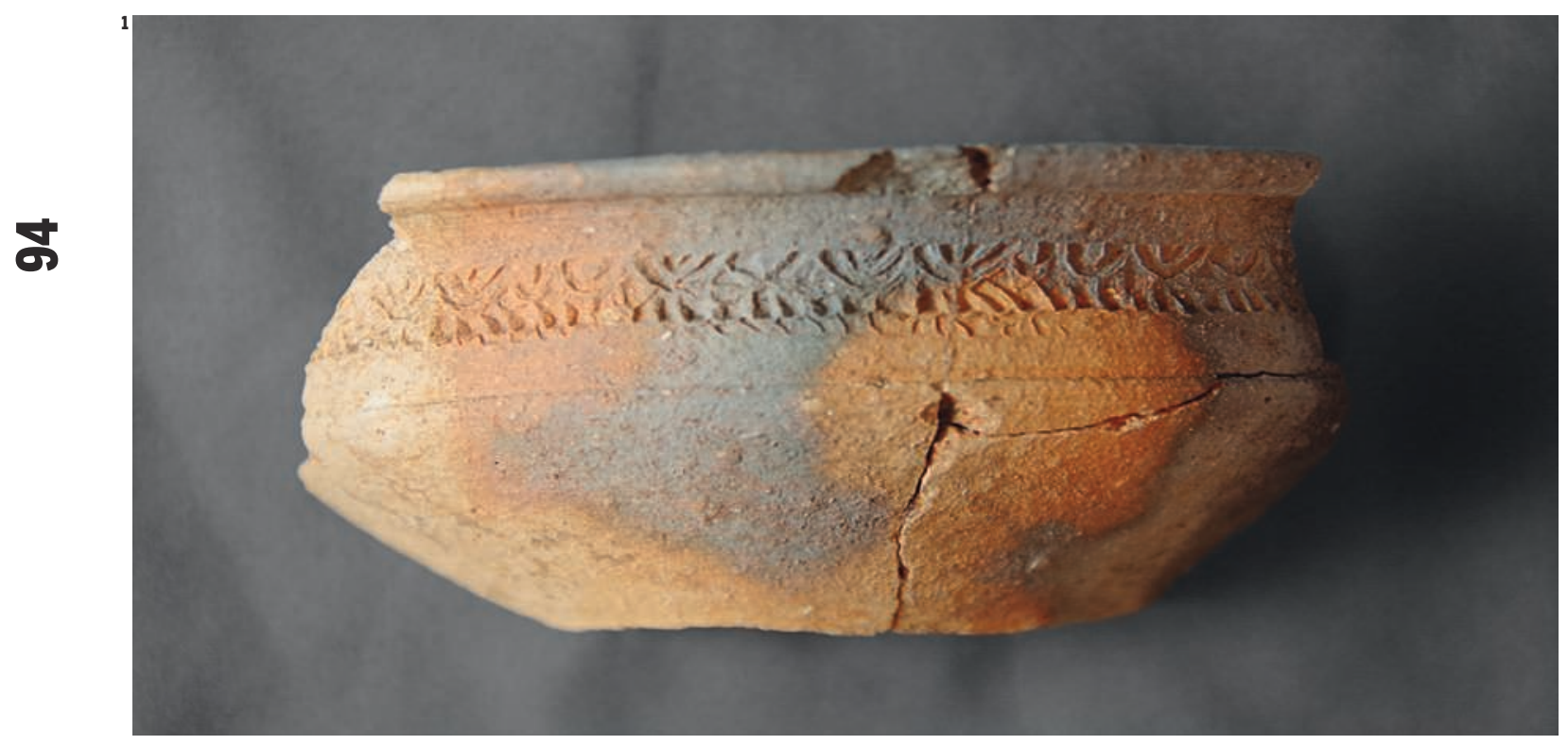

1. Coupelle à carène médiane du vil' siècle décorée d'un molette à motifs d'oves. Sa surface externe présente des taches gris-bleuté, stigmates des dépôts de cendres n'ayant pas permis la réoxydation (le Bourg).

2. Phénomène de réoxydation imparfaite des surfaces obtenu lors de la cuisson expérimentale de 2009, dans le four à double foyer (la Médecinerie).

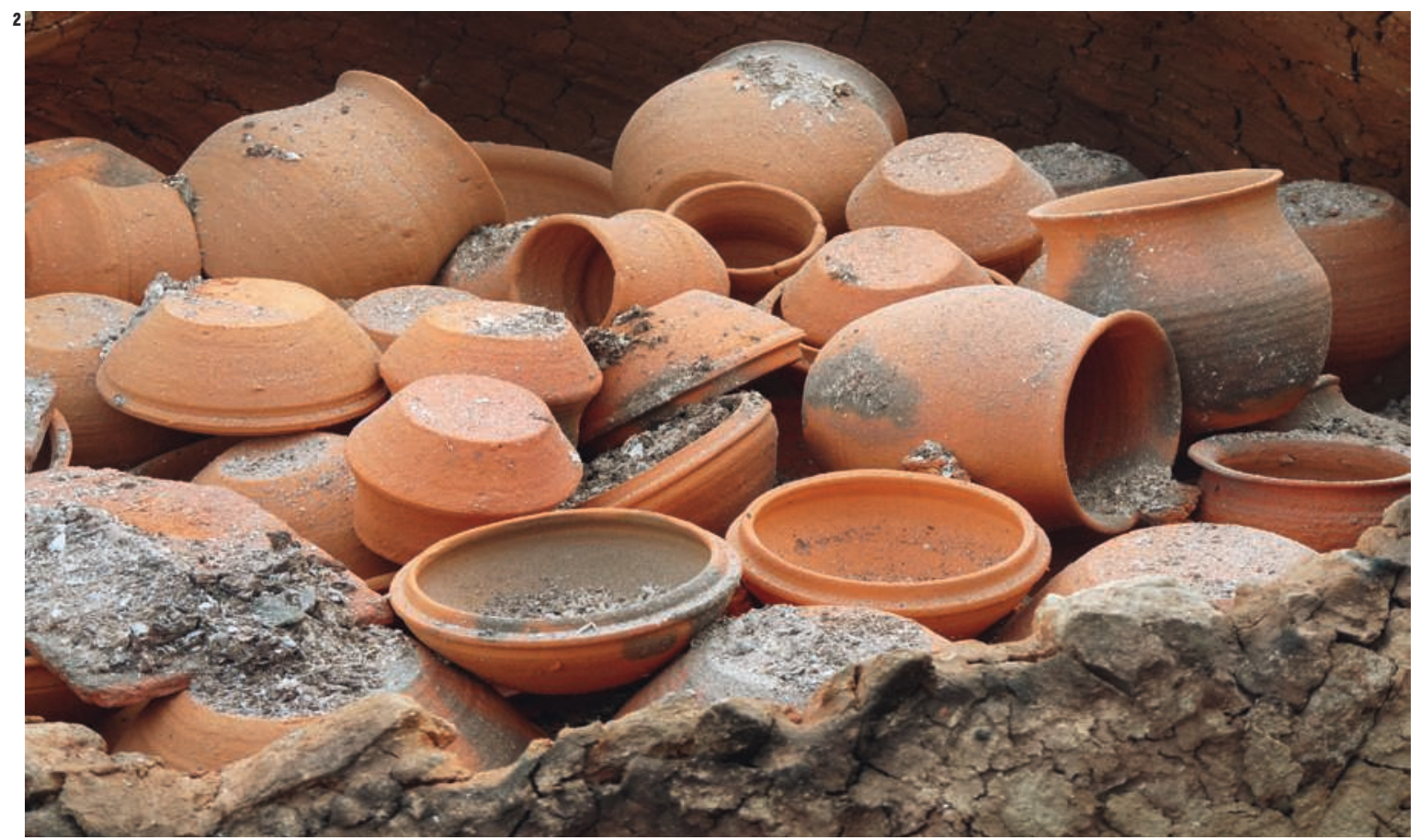


de la préparation de la pâte, le malaxage pouvant provoquer la formation de bulles d'air, mais aussi par la nature même de la matière première, notamment des argiles riches en calcaire.

Les phénomènes de surcuisson, avec parfois une perte totale de cohésion, sont rares, attestant d'une bonne maîtrise des températures de cuisson. À défaut de «mouton ", d'adhérence et de surfaces pseudo-grésées, il faut conclure à l'absence d'emballement du four. Les problèmes de souscuisson plus fréquents, notamment pour la deuxième moitié du VIII ${ }^{\mathrm{e}}$ siècle et le $\mathrm{IX}^{\mathrm{e}}$ siècle, sont interprétés quant à eux comme un enfournement imparfait du vase dans un secteur situé en dehors de la circulation de la chaleur. Afin de mieux apprécier la qualité de l'argile locale, il convient enfin de rappeler les expérimentations menées avec l'argile prélevée in situ qui permet, malgré des conditions climatiques peu favorables, notamment de fortes pluies en fin de cuisson, de réaliser des productions viables. Ce constat permet d'envisager des ratés de cuisson résultant plus vraisemblablement de problèmes de séchage (par ailleurs soulignés par les potiers modernes) et/ou d'enfournement (Jesset, 2008, p. 177).

Les structures de séchage peuvent être évoquées suivant un raisonnement par défaut. De fait, les compétences des potiers de Saran s'étendent à la production de terres cuites architecturales. Sur les tegulae principalement, on observe sur la surface supérieure diverses empreintes, notamment d'animaux (domestiques ou sauvages). Ces témoignages d'une exposition extérieure à même le sol dans des aires de séchage sans doute couvertes sont absents sur les céramiques. Ce constat permet de supposer des aménagements spécifiques en hauteur et protégés des aléas extérieurs. Les plans des bâtiments annexes aux fours peuvent en constituer les infrastructures (Jesset, 2015).

\section{Références bibliographiques}

Berger G., Desprez N., 1969, Carte géologique de la France (1/50 ooo), feuille Orléans (363): Notice explicative, Orléans, Bureau de recherches géologiques minières, $8 \mathrm{p}$.

Bouillon J., CAPron F. (dir.), 2015, Loiret, Saran, Ancienne route de Chartres, au lieu-dit «La Guignace» - (Zone sud et zone nord): Une extension nord au complexe artisanal potier de Saran "La Médecinerie » $\left(V I^{e}-X^{e}\right.$ siècle), rapport d'opération, Inrap Centre Île-de-France, 2 vol., 709 et $598 \mathrm{p}$.

Chapelot J., 1969, Saran, un centre de fabrication de la terre-cuite à la fin de l'époque mérovingienne et au début de l'époque carolingienne, rapport de fouille archéologique, SRA Centre, 8 p.

Dessandier D., JÉzÉQUel P. (dir.), 2001, Comparaison des propriétés minéralogiques et pétrographiques d'échantillons d'argiles crues et cuites concernant le site de «la ZAC des Vergers » à Saran (45), ANA/CMI/NT/o1/121, BRGM, 8 p. et annexe.

GuAdagnin R., 200o, Fosses, vallée de l'Ysieux mille ans de production céramique en Île-de-France, Caen, Publications du CRAHM, $367 \mathrm{p}$

Jesset S. (dir.), 2001, La «ZAC des Vergers ", Saran (Loiret), 45.302.008. AH, DFS, Orléans, SRA Centre, vol. 1 et 2, 267 p.; vol. 3 (annexes), 193 p.

JESSET S. (DIR.), 2007, «Étude d'un lot de céramiques du haut Moyen Âge découvert dans le réseau de l'Abîme (Orléans-la-Source) », Revue archéologique du Loiret, $\mathrm{n}^{\circ}$ 30-31, p. 35-54.

JESSET S., 2008, «Au sujet d'une cuisson expérimentale de céramique sur le site du haut Moyen Âge de Saran, "ZAC des Vergers" ", Revue archéologique du Loiret, $\mathrm{n}^{\circ}$ 32, p. 173-180.
JeSSET S., 2013, " Héritage et traditions décoratives ", in Husi P. (dir.), La céramique du haut Moyen Âge dans le Centre-ouest de la France : de la chronotypologie aux aires culturelles, $49^{\mathrm{e}}$ Supplément à la Revue archéologique du Centre de la France, Tours, FERACF, p. 211-220.

JESSET S., 2015, « Les ateliers de potiers du haut Moyen Âge autour d'Orléans (Loiret) : caractérisation, organisation et production ", in THUILliER F., LOUIS É. (éd.), Tourner autour du pot... Les ateliers de potiers médiévaux $d u V^{e}$ au XII siècle dans l'espace européen, Actes du colloque de Douai du 5 au 8 octobre 2010, Caen, Presses universitaires de Caen, Collection Publications du Craham, p. 227-247.

Laurent-DehecQ A., Payet-Gay K., Fencke E. (dir.), à paraître, Saran, « la Hutte, le Mesnil » (45), ZAC Portes du Loiret, Zones $C$ et E, rapport d'opération, CD 45, SRA Centre.

LEJAULT C., à paraître, «Étude du mobilier céramique », in LAURENT-DEHECQ et al., à paraître.

Millet S., 2015, « L'apport des expérimentations et des analyses connexes : l'exemple de Saran 2000/2009 (Loiret)», in Thuillier F., Louis É. (éd.), Tourner autour du pot... Les ateliers de potiers médiévaux $d u V^{e}$ au XII siècle dans l'espace européen, Actes du colloque de Douai du 5 au 8 octobre 2010, Caen, Presses universitaires de Caen, Collection Publications du Craham, p. 623-633.

Pauly S., 2008, Saran (Loiret), Analyses pétrographiques et physico-chimiques de la production de l'atelier des Vergers, Rapport d'étude, Nantes, Laboratoire Arc'Antique, 35 p. 